\title{
HH production at the High Luminosity LHC Prospects with the CMS experiment
}

\section{Luca Cadamuro* on behalf of the CMS Collaboration}

University of Florida

E-mail: luca.cadamuro@cern.ch

The exploration of the scalar potential and of its role in the spontaneous breaking of electroweak symmetry is one of the main goals of the physics program of the Large Hadron Collider. The study of Higgs boson pair production is particularly important in this context, as it gives a direct experimental access to the shape of the scalar potential itself. Because of the small cross section, this process is particularly challenging and its experimental study will greatly benefit of the large datasets collected at the High-Luminosity LHC (HL-LHC). This document describes the main challenges for the CMS experiment, the upgrade program to address them, and the prospects for Higgs boson pair production studies at the HL-LHC.

The 39th International Conference on High Energy Physics (ICHEP2018)

4-11 July, 2018

Seoul, Korea

${ }^{*}$ Speaker. 


\section{Introduction}

The exploration of the scalar sector of the standard model of particle physics (SM) is one of the main goals of the LHC program. The determination of the shape of the scalar potential is of special importance, as it directly determines how the electroweak symmetry breaking (EWSB) occurs and what are the properties of the Higgs boson. In the SM, a direct link exists between the parameter that determines such shape and the strength of the Higgs boson self coupling, $\lambda_{\mathrm{HHH}}$, that is experimentally accessible from the study of Higgs boson pair $(\mathrm{HH})$ production.

At tree level, this process occurs at the LHC through two heavy quark loop diagrams involving either the separate emission of two Higgs bosons, or the Higgs boson self-interaction itself. Their destructive interference results in a small cross section of $\sigma_{\mathrm{HH}}=36.69_{-4.9 \%}^{+2.1 \%}$ at $\sqrt{s}=14 \mathrm{TeV}$, computed at NNLO+NNLL QCD precision with finite top quark mass effects [1]. The tiny cross section makes the HH signal particularly elusive at the LHC.

\section{The HL-LHC and the upgraded CMS detector}

The High-Luminosity LHC (HL-LHC) will constitute an unprecedented opportunity for $\mathrm{HH}$ exploration. With an integrated luminosity of $3 \mathrm{ab}^{-1}$ or more collected by the end of HL-LHC operations, the CMS experiment [2] has the potential to precisely study $\mathrm{HH}$ production and measure $\lambda_{\mathrm{HHH}}$. HL-LHC operations are planned to start in 2025 after an upgrade of the machine that will result in an increase of about a factor of 5 of the instantaneous luminosity over the design values, and produce on average 140 to 200 simultaneous pp interactions per bunch crossing (pileup).

To maintain the performance in such a harsh collision environments, the CMS experiment is preparing and ambitious upgrade program of the detector [3]. The bandwidth of the trigger and data acquisition systems will be increased by about a factor of 7 with respect to the current values. The L1 trigger will also have an increased latency of $12.5 \mu \mathrm{s}$ and feature inputs from the inner tracker, allowing for sophisticated reconstruction algorithms implemented at the hardware level. The forward calorimeter will be replaced with a radiation hard, high-granularity silicon calorimeter, with the capability to reconstruct tridimensional spatial information and provide timing information. A new inner tracker will be installed and will have an increased granularity, reduced material budget and extended coverage. Upgrades in the electronics are foreseen for the barrel electromagnetic calorimeter, and drift tubes and cathode strip chambers in the muon systems, and new muon detectors will be installed in the endcaps of the experiment. Finally, the introduction of a detector for precise timing measurements of minimum ionizing particles is under study to suppress the pileup contribution and improve the performance of physics object reconstruction.

\section{HH at the HL-LHC}

HH production results in a phenomenologically rich set of final states. The CMS experiment is pursuing a broad program of searches using data collected at $\sqrt{s}=13 \mathrm{TeV}$ during the so-called Run II in the decay channels bbbb, bb $\tau \tau$, bb $\gamma \gamma$, and bbVV ( $\mathrm{V}=\mathrm{W}, \mathrm{Z}$ using $\mathrm{VV} \rightarrow \ell v_{\ell} \ell^{\prime} v_{\ell^{\prime}}$ decays), and the HL-LHC will open up the possibility to study new rare but clean signatures such as bbZZ with $\mathrm{ZZ} \rightarrow 4 \ell$. All these searches will benefit from the upgrade program previously described. 


\subsection{Run II extrapolations}

A first set of extrapolations, summarised in Table 1, has been performed in the context of the European Committee for Future Accelerators (ECFA) workshop held in 2016 using the results obtained with the $2.3-2.7 \mathrm{fb}^{-1}$ dataset collected at $\sqrt{s}=13 \mathrm{TeV}$ in 2015 [4], Systematic uncertainties are assumed to be reduced according to the "S2 scenario" defined in [4].

\begin{tabular}{|c|c|c|c|c|c|c|}
\hline \multirow{2}{*}{ Channel } & \multicolumn{2}{|c|}{ Limits on $\mu=\sigma_{\mathrm{HH}} / \sigma_{\mathrm{HH}}^{\mathrm{SM}}$} & \multicolumn{2}{|c|}{ Significance (Z-value) } & \multicolumn{2}{|c|}{ Uncertainty on $\mu$} \\
\hline & Syst.+stat. (S2) & Stat. only & Syst.+stat. (S2) & Stat. only & Syst.+stat. (S2) & Stat. only \\
\hline $\mathrm{HH} \rightarrow \mathrm{bb} \gamma \gamma$ & 1.44 & 1.37 & 1.43 & 1.47 & 0.72 & 0.71 \\
\hline $\mathrm{HH} \rightarrow \mathrm{bb} \tau \tau$ & 5.2 & 3.9 & 0.39 & 0.53 & 2.6 & 1.9 \\
\hline $\mathrm{HH} \rightarrow \mathrm{bbVV}$ & 4.8 & 4.6 & 0.45 & 0.47 & 2.4 & 2.3 \\
\hline $\mathrm{HH} \rightarrow \mathrm{bbbb}$ & 7.0 & 2.9 & 0.39 & 0.67 & 2.5 & 1.5 \\
\hline
\end{tabular}

Table 1: 95\% CL upper limits on $\mu=\sigma_{\mathrm{HH}} / \sigma_{\mathrm{HH}}^{\mathrm{SM}}$, significance, and uncertainty on the signal strength $\mu$ for the four HH decay channels results extrapolated to a luminosity of $3000 \mathrm{fb}^{-1}$ [4].

These results highlight the need to explore and combine many channels to maximise the experimental reach for HH. Although a limited combined sensitivity to HH seems to be achieved, these projections are very conservative. More recent Run II results based on the 2016 dataset of $35.9 \mathrm{fb}^{-1}$ showed that the sensitivity may improve faster than expected from a simple scaling with the integrated luminosity, since larger datasets allow for more sophisticated analysis methods to be developed. An example is offered by the $\mathrm{HH} \rightarrow$ bb $\tau \tau$ decay channel, where the projected upper limit at the 95\% confidence level (CL) as a function of the integrated luminosity is shown in Fig. 1a. While a limit of 25 times the SM prediction was expected to be achieved with $100 \mathrm{fb}^{-1}$ in the most optimistic scenario (not strictly valid below $300 \mathrm{fb}^{-1}$ ), the Run II search [5] has achieved the same sensitivity using only $35.9 \mathrm{fb}^{-1}$, i.e. with a dataset about a factor of 3 smaller.

These observations bring new hope to the $\mathrm{HH}$ prospects and motivate the preparation of updated projections, that will appear in the fifth CERN Yellow Report.

\subsection{Highlights of updated results: $b b \tau \tau$}

Prospects in the bb $\tau \tau$ decay channel have been recently studied [6] to account for the improvements in analysis method and the upgraded detector impact. Both the gluon and the vector boson fusion production mechanisms have been investigated. While the cross section of the latter is about a factor of 20 smaller than the former, the forward jet signature, combined with the performance of the upgraded endcap calorimeter, allows for the reduction of background processes and thus increases the sensitivity. The expected 95\% CL upper limit corresponds to 1.7 (51) times the SM gluon (vector boson) fusion cross section prediction, resulting in a combined limit of 1.6 times the SM expected signal strength, for a significance ranging between 1.1 and $1.3 \sigma$ depending on the assumptions made on the systematic uncertainties

\subsection{Beyond the SM}

The HL-LHC will also allow for the study of manifestation of physic beyond the SM (BSM) in $\mathrm{HH}$ production. The CMS Collaboration has studied the prospects for the search of massive scalar 
resonances produced in vector boson fusion and decaying to $\mathrm{HH}$ [7], a process expected in models with extra compact dimensions where the couplings to vector bosons are dominant, where the small cross section makes them currently unaccessible at the LHC. The search uses the characteristic forward jet signature, and the so-called "boosted objects" techniques to reconstruct the decays of high Lorentz boost Higgs bosons to b quarks as a single large radius jet $J$. The resonant signature of the two selected $J$ provides a good discrimination from the large multijet background. As shown in Fig. 1b, the existence of such processes could be seen using the large HL-LHC dataset.

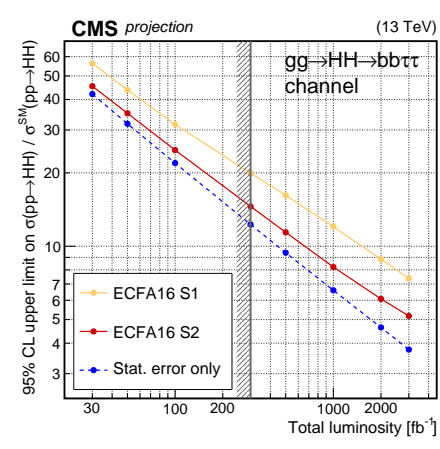

(a)

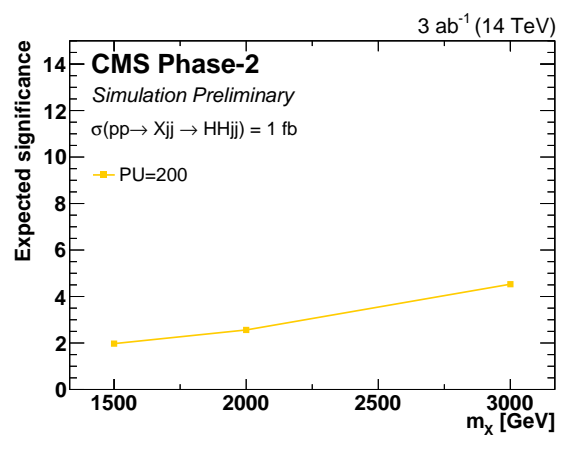

(b)

Figure 1: (a) Projected 95\% CL upper limit in the bb $\tau \tau$ decay channel from the extrapolation of early Run II results [6]. More recent Run II results based on the full 2016 dataset have shown that the projected values are very conservative. (b) Projected significance for the search of resonant $\mathrm{HH}$ production in vector boson fusion [7].

\section{Conclusions}

The exploration of Higgs boson pair production is crucial towards our understanding of EWSB and its role in the SM, and the HL-LHC will represent an unprecedented possibility for HH studies. An ambitious upgrade program is in preparation to maintain and improve the CMS detector performance at the HL-LHC. Current projections, based on the extrapolation of early Run II results, show that a comprehensive exploration of several $\mathrm{HH}$ decay channels will be essential to maximise the sensitivity, but have already been shown to be conservative by more recent results. Updated projections are in preparation and will appear in the fifth CERN Yellow Report.

\section{References}

[1] Grazzini, M., Heinrich, G., Jones, S. et al. J. High Energ. Phys. (2018) 2018: 59.

[2] CMS Collaboration, JINST 3 S08004 (2008).

[3] CMS Collaboration, CERN-LHCC-2015-010. CDS ID: 2020886

[4] CMS Collaboration, CMS-PAS-FTR-16-002. CDS ID: 2266165

[5] CMS Collaboration, Phys. Lett. B 778 (2018) 101.

[6] CMS Collaboration, CERN-LHCC-2017-023. CDS ID: 2293646

[7] CMS Collaboration, CMS-PAS-FTR-18-003. CDS ID: 2628598 